\title{
Genotyping and zoonotic potential of Enterocytozoon bieneusi in cattle farmed in Hainan Province, the southernmost region of China
}

Xin-Li Zheng ${ }^{1, a}$, Huan-Huan Zhou ${ }^{2,3,4, a}$, Gangxu Ren ${ }^{2,3,4, a}$, Tian-Ming Ma ${ }^{2,3,4}$, Zong-Xi Cao ${ }^{1}$, Li-Min Wei ${ }^{1}$, Quan-Wei Liu ${ }^{1}$, Feng Wang ${ }^{1}$, Yan Zhang ${ }^{1}$, Hai-Long Liu ${ }^{1}$, Man-Ping Xing ${ }^{1}$, Li-Li Huang ${ }^{1}$, Zhe Chao ${ }^{1, *}$, and Gang Lu ${ }^{2,3,4, *}$

${ }^{1}$ Institute of Animal Science and Veterinary Medicine, Hainan Academy of Agricultural Sciences, 571100 Haikou, PR China

${ }^{2}$ Key Laboratory of Tropical Translational Medicine of the Ministry of Education, Hainan Medical University, 571199 Haikou, PR China

${ }^{3}$ Department of Pathogenic Biology, Hainan Medical University, 571199 Haikou, Hainan, PR China

${ }^{4}$ Hainan Medical University-The University of Hong Kong Joint Laboratory of Tropical Infectious Diseases, Hainan Medical University, 571199 Haikou, Hainan, PR China

Received 7 October 2020, Accepted 9 November 2020, Published online 24 November 2020

\begin{abstract}
Enterocytozoon bieneusi is an intestinal pathogen that infects a wide range of species, including humans. Cattle constitute an important host for E. bieneusi; however, there is a scarcity of information on the prevalence and genotyping of E. bieneusi in cattle in the Hainan Province of China. In this study, PCR analysis of 314 fecal samples from cattle in six cities of Hainan was performed for genotype identification. The average prevalence of E. bieneusi in these animals was $9.9 \%(31 / 314)$, and ranged from $0.0 \%$ (0/12) to $20.5 \%$ (8/39). Five known genotypes - EbpC $(n=14)$, BEB4 $(n=12), \mathrm{J}(n=2), \mathrm{I}(n=1)$, and CHG5 $(n=1)-$ and a novel genotype: HNC-I $(n=1)-$ were identified. Genotypes EbpC and HNC-I were placed in zoonotic Group 1, and the remaining four genotypes (BEB4, J, I, and CHG5) were placed in Group 2. Since 93.5\% of the genotypes found in the cattle (29/31) (EbpC, BEB4, J, and I) have previously been found in humans, these genotypes are probably involved in the transmission of microsporidiosis to humans.
\end{abstract}

Key words: Enterocytozoon bieneusi, Cattle, Genotyping, Hainan (China).

Résumé - Génotypage et potentiel zoonotique d'Enterocytozoon bieneusi chez les bovins élevés dans la province de Hainan, la région la plus au sud de la Chine. Enterocytozoon bieneusi est un pathogène intestinal qui infecte un large éventail d'espèces, y compris les humains. Le bétail constitue un hôte important pour E. bieneusi, mais les informations sur la prévalence et le génotypage d'E. bieneusi chez les bovins de la province de Hainan en Chine sont rares. Dans cette étude, une analyse PCR de 314 échantillons fécaux provenant de bovins dans six villes de Hainan a été réalisée pour l'identification du génotype. La prévalence moyenne d'E. bieneusi chez ces animaux était de $9,9 \%(31 / 314)$, et variait de $0,0 \%(0 / 12)$ à 20,5\% (8/39). Cinq génotypes connus, EbpC $(n=14)$, BEB4 $(n=12), \mathrm{J}(n=2), \mathrm{I}(n=1)$ et CHG5 $(n=1)$, et un nouveau génotype, HNC-I $(n=1)$, ont été identifiés. Les génotypes EbpC et HNC-I sont placés dans le groupe zoonotique 1, et les quatre génotypes restants (BEB4, J, I et CHG5) sont placés dans le groupe 2. Puisque 93,5 \% (29/31) (EbpC, BEB4, J et I) des génotypes trouvés chez les bovins ont déjà été trouvés chez l'homme, ces génotypes sont probablement impliqués dans la transmission de la microsporidiose à l'homme.

\section{Introduction}

Enterocytozoon bieneusi, a zoonotic intestinal pathogen, infects a wide range of species worldwide [20, 24]. Microsporidiosis occurs through the ingestion of infectious spores of E. bieneusi through contaminated soil, feces, surfaces, water, as well as by improper farming practices, such as using untreated animal manure as fertilizer directly on open crops or tillage land [20]. Enterocytozoon bieneusi has received considerable attention due to its known propensity to cause both water- and food-borne outbreaks of illness [44].

Sequence analysis of the internal transcribed spacer (ITS) region of the ribosomal RNA (rRNA) gene has revealed more than 500 genotypes (142 in humans, of which 49 were also

*Corresponding authors: chaozhe. cn@126. com; luganghn@163.com

${ }^{a}$ These authors contributed equally to this work. 
Table 1. ITS genotypes of Enterocytozoon bieneusi of natural infection identified in cattle worldwide.

\begin{tabular}{|c|c|c|c|}
\hline Country & Positive/examined (\%) & Genotypes $^{\mathrm{a}}(n)$ & Ref. \\
\hline Algeria & $11 / 102(10.8)$ & $\begin{array}{l}\text { BEB4 (4), BEB6 (2), BEB3 (1), I (1), J (1), PtEb XI (1), mixed } \\
\text { (1) }\end{array}$ & {$[2]$} \\
\hline Argentina & $10 / 70(14.3)$ & BEB4 (1); I (2), J (4); EbpC (1); BEB10 (1); D (1) & {$[5]$} \\
\hline Australia & $49 / 471(10.4)$ & I (18), J (14), BEB4 (6), TAR_fc2 (6), TAR_fc1 (1), TAR_fc3 (1) & [48] \\
\hline Brazil & $79 / 452(17.5)$ & $\begin{array}{l}\text { I (35), BEB8 (23), BEB4 (7), BEB13 (7),BEB12 (5), D (4), } \\
\text { BEB11 (3), EbpA (1), BEB14-BEB17 (1 each) }\end{array}$ & [4] \\
\hline China & $1817 / 10504(17.3)$ & $\begin{array}{l}\text { J (904); I (519); BEB4 (151), BEB6 (31), O (27), CM8 (18), } \\
\text { COS-I (14), EbpC (14), CHN3 (14), D (13), CHN1 (11), CGC3 } \\
\text { (11), CGC2 (8), CHC8 (7), CGC1 (6), CHN4 (6), CS-4 (6), } \\
\text { Type IV (5), CM19 (5), BEB10 (3), CHG2 (2), CHG3 (2), } \\
\text { CHN-DC1 (2), CHN-DC2 (2), CHN-DC3 (2), G (2), NECA1- } \\
\text { NECA5, CHC1-CHC7, CHC9-CHC17, N, BEB8, CD6, H, } \\
\text { CC4, CSX1-CSX2, CHN15, CM21, PN, and mixed (1 each) }\end{array}$ & $\begin{array}{c}{[10,12,13,19,23,26,} \\
37,38,41-43,46,47, \\
49,50]\end{array}$ \\
\hline Czech Republic & $37^{\mathrm{b}} / 240(15.4)$ & I (6) & [14] \\
\hline Germany & $10 / 88(11.4)$ & $\mathbf{I}(2) ; \mathbf{J}(4) ; \mathbf{F}(1) ; \mathbf{M}(1) ; \mathbf{N}(1), \mathbf{I} / \mathbf{J}(1)$ & {$[6,27]$} \\
\hline Iran & $48 / 256(18.8)$ & $\mathbf{D}(22), \mathbf{J}(9), \mathbf{M}(5)$ & [16] \\
\hline Korea & $80^{\mathrm{b}} / 538(14.9)$ & CEbE (3), CEbD (2), CEbB (2), CEbA (1), CEbF (1), CEbC (1) & {$[17]$} \\
\hline Portugal & $2 / 2^{\mathrm{c}}(100.0)$ & PtEbX (1), PtEbXI (1) & {$[22]$} \\
\hline South Africa & $9 / 50(18.0)$ & BEB4 (3); I (1); BEB3-like (4); D (1) & {$[1]$} \\
\hline United States & $706 / 3306(21.4)$ & $\begin{array}{l}\text { J (110); BEB4 (120); BEB2 (85); I (79), BEB1 (47); BEB8 (41); } \\
\text { BEB5 (8); BEB9 (6); BEB3 (6); Peru } 6 \text { (1); D (1), BEB7 (1), } \\
\text { Type IV (1) }\end{array}$ & {$[8,9,31-33,36]$} \\
\hline Slovakia & $2 / 100(2.0)$ & $\mathbf{I}(2)$ & {$[40]$} \\
\hline Thailand & $3 / 60(5.0)$ & D (3) & [39] \\
\hline
\end{tabular}

${ }^{a}$ The names of genotypes are from publications.

${ }^{\mathrm{b}}$ The number of genotypes is not consistent with the number of positives because only some E. bieneusi isolates were genotyped in the Czech Republic and Korea.

${ }^{\mathrm{c}}$ Only two isolates positive for E. bieneusi by microscopy after staining were genotyped in Portugal.

The genotypes previously found in humans are shown in bold.

identified in animals) [11, 20, 54]. Phylogenetic comparative analyses clustered all genotypes into eleven major genetic groups. Human cases have been reported to show infection with E. bieneusi genotypes from six groups, and more than $90 \%$ of human-pathogenic genotypes belonged to Group 1 or Group 2 [20, 54].

Thirty-eight studies from 14 countries have identified more than 80 genotypes in cattle, known carriers of E. bieneusi (Table 1). Among them, at least 17 genotypes (BEB4, BEB6, I, J, PtEb XI, EbpC, D, EbpA, M, Type IV, Peru 6, H, O, CS-4, CHN3, CHN4, and S7) have also been identified in humans [20]. Of the remaining 67 genotypes, 30 belonged to Group 1, and 27 belonged to Group 2, indicating the vital role of cattle in the epidemiology of E. bieneusi and their ability to transmit the pathogen to humans [20]. Therefore, cattle infected with E. bieneusi may pose a threat to public health.

In China, cattle farming and dairy products are important economic industries. Previous studies on E. bieneusi in cattle in China focused on inland cities and did not include assessments in Hainan Province, the southernmost region of China, where, local yellow cattle breeding is very popular. Here, we evaluated the prevalence, genetic characteristics, and zoonotic potential of E. bieneusi in cattle from six cities of Hainan Province.

\section{Materials and methods}

\section{Ethics statement}

The study was initiated after obtaining written informed consent for animal use by farm owners. All animal experiments were reviewed and approved by the Ethics Committee of Hainan Medical University.

\section{Fecal specimen collection}

In all, 314 fecal samples were gathered from 10 cattle farms in six cities of Hainan Province between March and December 2019 (Fig. 1 and Table 2). The cattle farms were selected based only on the owners' willingness to participate and the accessibility of animals for sampling. Samples were obtained from 30$50 \%$ of the total number of cattle on each farm. A sterile disposable latex glove was used to collect the fecal specimens immediately post defecation, and placed in individually labeled plastic bags. Cattle were divided into two groups: young aged $\leq 12$ months $(n=18)$ and adults aged $>12$ months $(n=296)$. Cattle were in good health at the time of sampling. Within $24 \mathrm{~h}$ of sampling, the labeled fecal bags were transported and stored in the laboratory at $4{ }^{\circ} \mathrm{C}$ and were processed within $48 \mathrm{~h}$. 


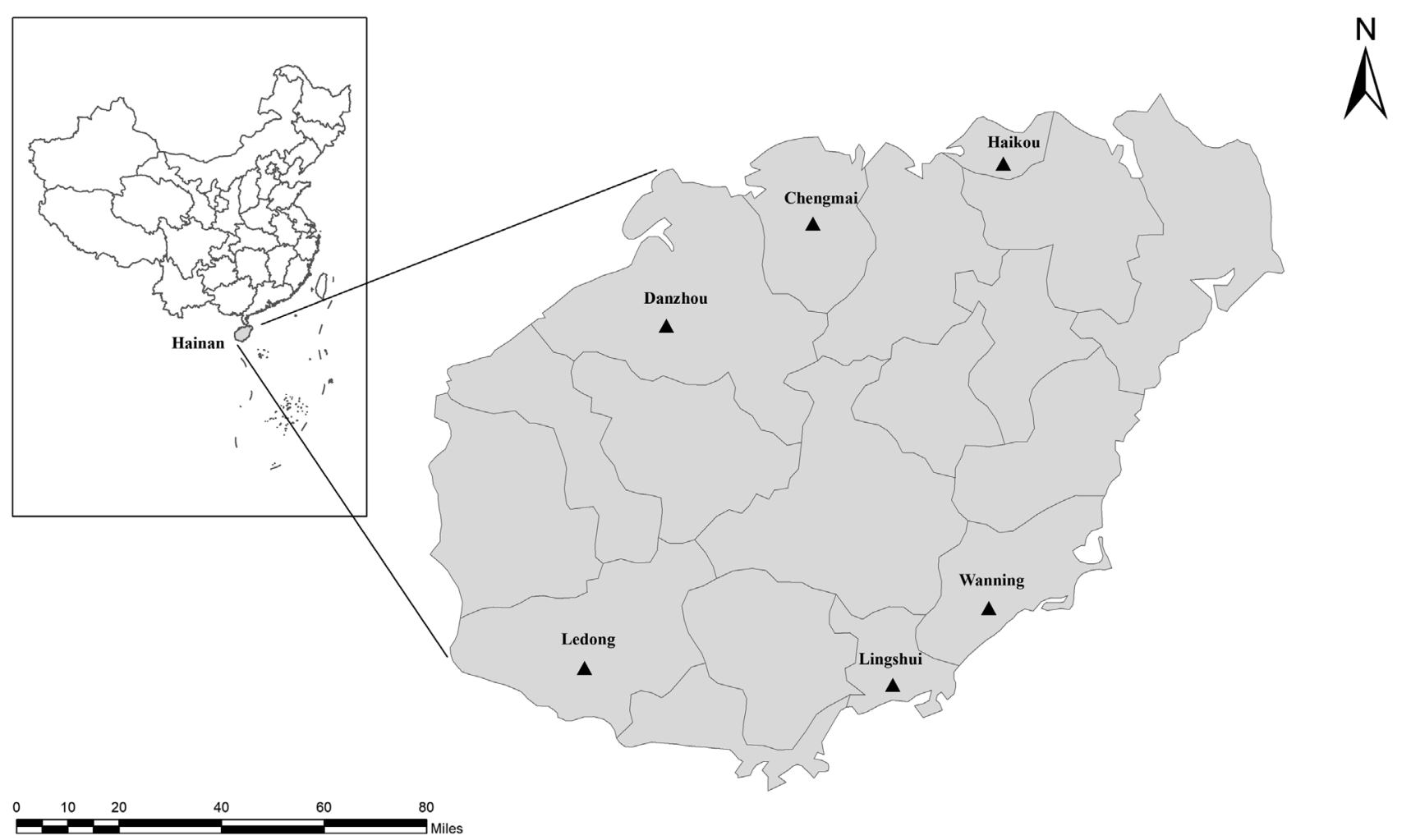

Figure 1. Specific locations where samples were collected in this study. $\mathbf{\Delta}$ : Sampling points.

Table 2. Prevalence and genotype distribution of E. bieneusi isolates in cattle in Hainan Province.

\begin{tabular}{lccc}
\hline Category & & Positive/examined (\%) & Genotype(s) $(n)$ \\
\hline Location & Farm 1 (Chengmai) & $2 / 13(15.4)$ & EbpC (2) \\
& Farm 2 (Chengmai) & $0 / 5(0.0)$ & J (2) \\
& Farm 3 (Chengmai) & $2 / 64(3.1)$ & EbpC (2), J (2) \\
& Subtotal (Chengmai) & $4 / 82(4.9)$ & EbpC (7), I (1) \\
& Farm 4 (Danzhou) & $8 / 39(20.5)$ & $/$ \\
& Farm 5 (Haikou) & $0 / 4(0.0)$ & EbpC (1) \\
& Farm 6 (Haikou) & $1 / 50(2.0)$ & $/$ \\
& Subtotal (Haikou) & $1 / 54(1.9)$ & $/$ \\
& Farm 7 (Ledong) & $0 / 12(0.0)$ & BEB4 (12) \\
& Farm 8 (Lingshui) & $0 / 26(0.0)$ & BEB4 (12) \\
& Farm 9 (Lingshui) & $12 / 70(17.1)$ & EbpC (4), CHG5 (1), HNC-I (1) \\
& Subtotal (Lingshui) & $12 / 96(12.5)$ & EbpC (3), I (1) \\
Age & Farm 10 (Wanning) & $6 / 31(19.4)$ & $4 / 18(22.2)$ \\
Total & $>12$ months & $27 / 296(9.1)$ & BEB4 (12), EbpC (11), J (2), CHG5 (1), HNC-I (1) \\
& $>12$ months & $31 / 314(9.9)$ & EbpC (14), BEB4 (12), J (2), I (1), CHG5 (1), HNC-I (1)
\end{tabular}

${ }^{\text {a }}$ All the cattle aged $\leq 12$ months were from farm 4 in Danzhou city.

\section{DNA extraction}

All fecal specimens were filtered through sieve in distilled water, followed by centrifugation at $1500 \times g$ for $10 \mathrm{~min}$. A QIAamp DNA stool mini kit (QIAgen, Germany) was used to isolate the genomic DNA of each processed specimen (approximately $200 \mathrm{mg}$ ), following the manufacturer's instructions. A total of $200 \mathrm{~mL}$ AE elution buffer was used to elute the DNA, followed by storage at $-20{ }^{\circ} \mathrm{C}$ before PCR analysis.

\section{Polymerase chain reaction (PCR) amplification}

Enterocytozoon bieneusi-specific nested primers and cycle parameters designed by Hamed Mirjalal were used to amplify 
Table 3. ITS genotypes of natural Enterocytozoon bieneusi infections identified in cattle in China.

\begin{tabular}{|c|c|c|c|}
\hline Regions & Positive/examined (\%) & Genotypes $(n)$ & Ref \\
\hline$\overline{\text { Gansu }}$ & $320 / 1414(22.6)$ & $\begin{array}{l}\text { J J (155), I (126), CGC3 (11), CGC2 (8), CGC1 (6), BEB4 (5), } \\
\text { CM19 (5), BEB10 (3), CM21 (1) }\end{array}$ & {$[43]$} \\
\hline \multirow[t]{2}{*}{ Guangdong } & $160 / 1440(11.1)$ & $\mathbf{I}(91), \mathbf{J}(60), \mathbf{D}(4), \mathbf{B E B} 4(3), \mathbf{E b p C}(2)$ & {$[43]$} \\
\hline & $61 / 388(15.7)$ & $\mathbf{J}(58), \mathbf{D}(4)$ & {$[10]$} \\
\hline Hebei and Tianjin & $202 / 1040(19.4)$ & $\begin{array}{l}\text { I (87), J (83), BEB4 (18), CHC8 (7), BEB6 (3), N (1), EbpC } \\
\text { (1), CHC6 (1), CHC7(1) }\end{array}$ & {$[12]$} \\
\hline \multirow[t]{2}{*}{ Henan } & $28 / 44(6.0)$ & $\mathbf{I}(16), \mathbf{J}(7), \mathbf{B E B 4}(5)$ & [23] \\
\hline & $33 / 277(11.9)$ & $\begin{array}{l}\text { BEB6 (10), COS-1 (6), I (6), CHG2, CHG3, J, CHC9, CHC10, } \\
\text { CHC11, CHC12, CHC13, CHC14, CHC15, and CHC16 } \\
\text { (1 each) }\end{array}$ & {$[46]$} \\
\hline Henan and Ningxia & $214 / 879(24.3)$ & $\begin{array}{l}\text { J (77), I (61), CM8 (18), BEB6 (17), BEB4 (15), EbpC (6), } \\
\text { COS-1 (5), EbpA (2), D (2), BEB8, CD6, CHC1-CHC5, } \\
\text { CHG2, CHG3, H, and O (1 each) }\end{array}$ & {$[19]$} \\
\hline \multirow[t]{3}{*}{ Heilongjiang } & $31 / 526(5.9)$ & $\begin{array}{l}\text { J (10), CS-4 (7), I (3), BEB4 (2), EbpC (3), G (1), NECA1 - } \\
\text { NECA5 (1 each) }\end{array}$ & {$[13]$} \\
\hline & $40 / 133(30.1)$ & $\begin{array}{l}\text { O (26), EbpA (2), J (2), I (2), CHN-DC1-CHN-DC3 (2 each), } \\
\text { BEB4 (1), D (1) }\end{array}$ & {$[50]$} \\
\hline & 93/321 (29.0) & BEB4 (22), J (40), I (31) & {$[38]$} \\
\hline Jiangsu & $177 / 1366(13.0)$ & J (144), I (26), BEB4 (11), Type IV (1), CHC17 (1) & {$[42]$} \\
\hline Jilin & $35 / 93(37.6)$ & CHN3 (14); CHN1 (10); J (9); I (8); CHN4 (2) & [47] \\
\hline Liaoning & $1 / 11(9.1)$ & $\mathbf{J}(1)$ & {$[13]$} \\
\hline Qingha and Yunnan & $10 / 57(17.5)$ & J (5), COS I (3), PN (1), BEB6 (1) & [49] \\
\hline \multirow[t]{2}{*}{ Shaanxi } & $39 / 198(19.7)$ & I (21), J (16), CHN1 (1), CSX1 (1) & {$[41]$} \\
\hline & $34 / 173(19.7)$ & $\mathbf{I}(19), \mathbf{J}(14), \operatorname{CSX} 2(1)$ & [41] \\
\hline \multirow[t]{2}{*}{ Shandong } & $21 / 673(3.12)$ & J (18), BEB4 (2), I (1) & [41] \\
\hline & $3 / 148(2.0)$ & $\mathbf{I}(1), \mathbf{J}(2)$ & {$[23]$} \\
\hline Shanghai & $214 / 809(26.5)$ & $\begin{array}{l}\text { J (145), BEB4 (63), CHN4 (4), Type IV (4), CHN15 (1), } \\
\text { mixed (1) }\end{array}$ & {$[37]$} \\
\hline Xinjiang & $85 / 514(16.5)$ & J (57), I (19), BEB4 (4), D (2), EbpC (2), CC4 (1) & {$[26]$} \\
\hline
\end{tabular}

The genotypes previously found in humans are shown in bold.

a $410 \mathrm{bp}$ sequence in the ITS region of the rRNA gene using TaKaRa Taq DNA Polymerase [25]. The PCR products were analyzed using $1.5 \%$ agarose gel electrophoresis, followed by GelRed (Biotium Inc., USA) staining.

\section{Nucleotide sequencing and analysis}

The sequence accuracy of all E. bieneusi-positive PCR products (sequenced by Sangon Biotech Co., Ltd., China) was confirmed through bidirectional sequencing and the sequencing of additional PCR products. The Basic Local Alignment Search Tool (BLAST) and ClustalX 1.83 were used to compare the published GenBank sequences with the ones identified in this study to identify the genotypes of E. bieneusi. Genotypes that were identical to the genotypes deposited in the GenBank database were given the first published name, and those that generated ITS sequences with a single nucleotide substitution/deletion/insertion were identified as novel genotypes based on the DNA sequencing of minimum two PCR products [30]. The samples were labeled in the order of appearance by adding roman numerals after HNC (Hainan Cattle). A $243 \mathrm{bp}$ part of the ITS region of the rRNA gene of E. bieneusi was used for naming reference, following the established nomenclature system [30].

\section{Phylogenetic analysis}

A neighbor-joining phylogenetic tree was built using Mega $\mathrm{X}$ software, and the Kimura-2-parameter model with 1000 replicates to evaluate the relationship between the novel ITS genotype and the known genotypes, and to confirm the gene group designation.

\section{Statistical analysis}

Fisher's exact test and a Chi-square test were used to evaluate the difference in infection rates among different locations and ages, respectively, using SPSS v22.0 (IBM Corp., USA). A $p$-value $<0.05$ was regarded as statistically significant.

\section{Nucleotide sequence accession numbers}

The GenBank database accession number of the identified nucleotide sequence was MT193626.

\section{Results and discussion}

Of the 314 fecal samples, 31 (9.9\%) were E. bieneusipositive, based on sequence analysis of the ITS region of the 


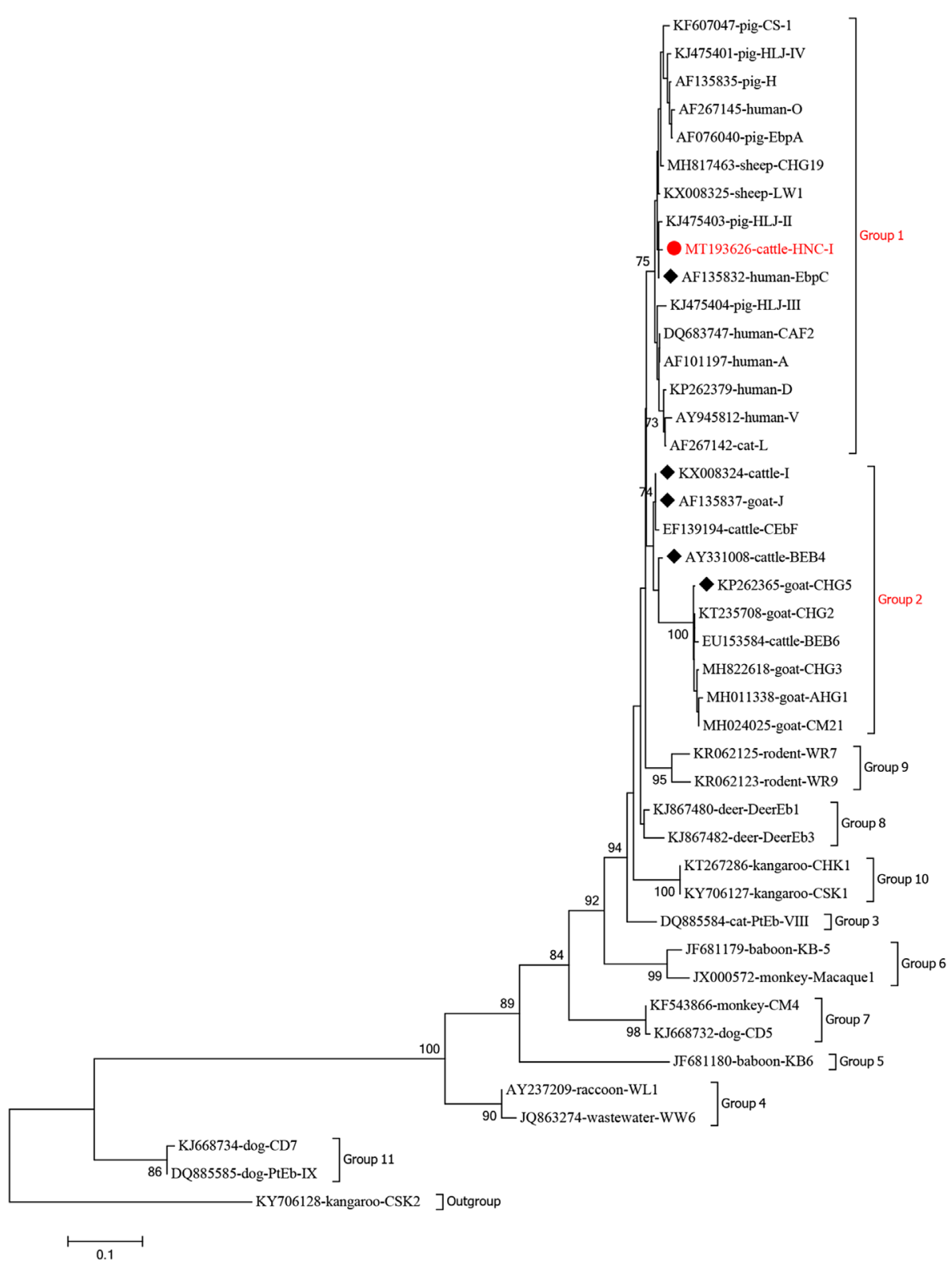

Figure 2. Phylogenetic tree based on neighbor-joining (N-J) analysis of ITS sequences. Phylogenetic relationships between the E. bieneusi genotypes identified in cattle here and other known genotypes deposited in GenBank were inferred by an N-J analysis of ITS sequences based on genetic distance by the Kimura two-parameter model. The numbers on the branches are percent bootstrapping values from 1000 replicates. Each sequence is identified by its accession number, host origin, and genotype designation. Enterocytozoon bieneusi genotype CSK2 (KY706128) was used as the outgroup. The squares and triangles filled in black indicate novel and known genotypes identified in this study, respectively.

rRNA gene. A significant difference in the rate of occurrence of E. bieneusi was observed in cattle from the six cities $(p<0.05)$, with $20.5 \%(8 / 39)$ in Danzhou, 19.4\% (6/31) in Wanning, $12.5 \%$ (12/96) in Lingshui, $4.9 \%$ (4/82) in Chengmai, $1.9 \%$ $(1 / 54)$ in Haikou, and an absence of this parasite (0/12) in Ledong (Table 2).

Since the first report of E. bieneusi in calves in Germany, there have been 38 published epidemiological reports on E. bieneusi conducted in 14 countries, and the average infection rates in these countries range from $2.0 \%$ to $21.4 \%$ (Table 1 ). The infection rate of E. bieneusi, based on cattle from 16 provinces of China, falls in the range of $2.0-37.6 \%$ (Table 3). This study reports the occurrence of E. bieneusi in cattle from Hainan Province. The differences in prevalence might be related to the sensitivity and specificity of detection methods, the health status of hosts, the experimental design, the overall sample size, animal practices, and so on. Like in other animals and humans, age appears to be a significant factor affecting the occurrence of E. bieneusi in cattle [51]. In the present study, the prevalence of E. bieneusi was $22.2 \%$ (4/18) in young animals $\leq 12$ months and 9.1\% (27/296) in adult animals $>$ 12 months. Although the infection rates in calves were higher than those in adults, the differences were not significant $\left(\chi^{2}=1.966, p>0.05\right)$ (Table 2$)$. A study by Ma et al. revealed 
E. bieneusi infection rates in juveniles, post-weaned calves, preweaned calves, and adults of $4.5 \%$ (6/134), 7.7\% (8/104), $10 \%$ (1/10), and 3.9\% (13/332), respectively [23]. Similarly, da Fiuza et al. reported that pre-weaned calves $(27.6 \%, 21 / 76)$ and post-weaned calves $(28.8 \%, 44 / 153)$ showed a higher rate of prevalence of E. bieneusi compared with heifers $(14.1 \%$, 12/85) and adults $(1.4 \%, 2 / 138)$ [4]. Meanwhile, Li et al. showed that calves aged $<3$ months $(29.3 \%, 127 / 434)$ and 3-12 months $(23.9 \%, 63 / 264)$ had higher infection rates than juveniles and adults $(13.3 \%, 24 / 181)$ [16]. In accordance with these results, it was supposed that age was negatively correlated with the prevalence of $E$. bieneusi in cattle, probably due to the underdeveloped immune systems of the young animals.

Here, we identified one novel genotype (HNC-I) and five known genotypes (EbpC, BEB4, J, I, and CHG5). The novel genotype showed high similarity to genotype EbpC (AF076042), with one base variation at position 237 $(\mathrm{C} \rightarrow \mathrm{T})$. Out of the six genotypes, the most prevalent genotype was EbpC (14 specimens), which was found in four of the six locations, followed by BEB4 (12 specimens), but this genotype was only found in Wanning. Genotype $\mathbf{J}$ was found in two cattle from Chengmai. The remaining three genotypes I, CHG5, and HNC-I were found in a single specimen, with the former from Danzhou and the latter two from Wanning. These results differed from those reported from the other regions of China. For example, in Gansu, Guangdong, Henan, Ningxia, Jiangsu, Shaanxi, and Xinjiang provinces, genotypes $\mathbf{J}$ and I were reported to be the dominant genotypes, and in Heilongjiang, genotype $\mathrm{O}$ was dominant (Table 3). Meanwhile, region-specific difference in genotype constitutions of E. bieneusi can also be observed in cattle in some studies, such as genotype $D$ in Iran [16]. Therefore, the genotype distributions of E. bieneusi in cattle differed by region, but the reason behind this phenomenon is unclear.

In the present study, human-pathogenic genotypes EbpC, BEB4, J and I were observed with high occurrence (93.5\%, 29/31). Genotype EbpC has been detected in humans, such as in cancer patients in Iran [25], in immunocompetent patients in the Czech Republic [29], in children in Peru and China [3, 45], and in HIV-positive patients in Peru, China, Iran, Thailand, and Vietnam [7, 18, 21, 25, 35, 41]. It was also found in more than 15 animal species and water samples [20]. Likewise, genotypes BEB4, J, and I were also found in humans [28, 47], nonhuman primates [15, 46], and other animals [20], and they have been documented in cattle (Table 1). This suggests that cattle infected with genotypes EbpC, BEB4, J, and I may facilitate transmission to other animals and humans.

The remaining genotype CHG5 and the novel genotype HNC-I were first identified in cattle here. Genotype CHG5 has been reported in goats with a wide distribution in China $[34,53]$. We also observed this genotype in the Asiatic brush-tailed porcupines in Hainan Province [52]. Thus, the detection of the same genotype (CHG5) in multiple species (cattle, goats, and rodents) in the same region (Hainan, China) suggests a vast host range along with the possibility of crossspecies transmission among cattle, goats and rodents.

The phylogenetic analysis revealed that EbpC and HNC-I, identified in this study, were divided into zoonotic Group 1, whereas genotypes BEB4, J, I, and CHG5 belong to Group 2
(Fig. 2). In total, $94.0 \%$ (79/84) of the genotypes identified in cattle clustered into Group 1 or 2 (except for genotypes CX1, CX2, TAR_fc3, CAM2, and S7) [20]. These findings suggest that $E$. bieneusi-infected cattle represent a potential threat to humans.

\section{Conclusion}

This study is the first evaluating the infection rates, genotype characteristics, and zoonotic potential of E. bieneusi in cattle from Hainan Province. Our results revealed a prevalence rate of 9.9\% (31/314) for E. bieneusi within five of six cities in Hainan, China. We identified five known genotypes and a novel genotype. Genotype EbpC and novel genotype HNC-I were grouped into zoonotic Group 1, while genotypes BEB4, J, I and CHG5 were placed in Group 2. The observed high occurrence $(93.5 \%, 29 / 31)$ of zoonotic genotypes (EbpC, BEB4, $\mathrm{J}$, and I) emphasizes the possible role of cattle in the transmission of $E$. bieneusi to humans, which requires further investigations to reduce the threats posed by these animals to public health.

Acknowledgements. This work was supported by the Young Talents Science and Technology Innovation Project of the Hainan Association for Science and Technology (QCXM201802); Hainan major science and technology project (ZDKJ2016017-01); the Innovation Research Team Project of the Hainan Natural Science Foundation (2018CXTD340); the National Natural Science Foundation of China (No. 81672072 and No. 81760378), and the Graduate Student Innovation Foundation of colleges and universities of Hainan Province, 2019 (Hys2019-287). The funders had no role in the study design, data collection, data interpretation, or the decision to submit the work for publication.

\section{References}

1. Abu Samra N, Thompson PN, Jori F, Zhang H, Xiao L. 2012. Enterocytozoon bieneusi at the wildlife/livestock interface of the Kruger National Park, South Africa. Veterinary Parasitology, 190(3-4), 587-590.

2. Baroudi D, Khelef D, Hakem A, Abdelaziz A, Chen X, Lysen C, Roellig D, Xiao L. 2017. Molecular characterization of zoonotic pathogens Cryptosporidium spp., Giardia duodenalis and Enterocytozoon bieneusi in calves in Algeria. Veterinary Parasitology Regional Studies Reports, 8, 66-69.

3. Cama VA, Pearson J, Cabrera L, Pacheco L, Gilman R, Meyer S, Ortega Y, Xiao L. 2007. Transmission of Enterocytozoon bieneusi between a child and guinea pigs. Journal of Clinical Microbiology, 45(8), 2708-2710.

4. da Silva Fiuza VR, Lopes CW, de Oliveira FC, Fayer R, Santin M. 2016. New findings of Enterocytozoon bieneusi in beef and dairy cattle in Brazil. Veterinary Parasitology, 216, 46-51.

5. Del Coco VF, Córdoba MA, Bilbao G, de Almeida Castro P, Basualdo JA, Santín M. 2014. First report of Enterocytozoon bieneusi from dairy cattle in Argentina. Veterinary Parasitology, 199(1-2), 112-115.

6. Dengjel B, Zahler M, Hermanns W, Heinritzi K, Spillmann T, Thomschke A, Löscher T, Gothe R, Rinder H. 2001. Zoonotic potential of Enterocytozoon bieneusi. Journal of Clinical Microbiology, 39(12), 4495-4499.

7. Espern A, Morio F, Miegeville M, Illa H, Abdoulaye M, Meyssonnier V, Adehossi E, Lejeune A, Cam PD, Besse B, 
Gay-Andrieu F. 2007. Molecular study of microsporidiosis due to Enterocytozoon bieneusi and Encephalitozoon intestinalis among human immunodeficiency virus-infected patients from two geographical areas: Niamey, Niger, and Hanoi, Vietnam. Journal of Clinical Microbiology, 45(9), 2999-3002.

8. Fayer R, Santín M, Trout JM. 2007. Enterocytozoon bieneusi in mature dairy cattle on farms in the eastern United States. Parasitology Research, 102(1), 15-20.

9. Fayer R, Santin M, Macarisin D. 2012. Detection of concurrent infection of dairy cattle with Blastocystis, Cryptosporidium, Giardia, and Enterocytozoon by molecular and microscopic methods. Parasitology Research, 111(3), 1349-1355.

10. Feng Y, Gong X, Zhu K, Li N, Yu Z, Guo Y, Weng Y, Kváč M, Feng Y, Xiao L. 2019. Prevalence and genotypic identification of Cryptosporidium spp., Giardia duodenalis and Enterocytozoon bieneusi in pre-weaned dairy calves in Guangdong, China. Parasites \& Vectors, 12(1), 41.

11. Gong B, Yang Y, Liu X, Cao J, Xu M, Xu N, Yang F, Wu F, Li B, Liu A, Shen Y. 2019. First survey of Enterocytozoon bieneusi and dominant genotype Peru6 among ethnic minority groups in southwestern China's Yunnan Province and assessment of risk factors. PLoS Neglected Tropical Diseases, 13(5), e0007356.

12. Hu S, Liu Z, Yan F, Zhang Z, Zhang G, Zhang L, Jian F, Zhang S, Ning C, Wang R. 2017. Zoonotic and host-adapted genotypes of Cryptosporidium spp., Giardia duodenalis and Enterocytozoon bieneusi in dairy cattle in Hebei and Tianjin, China. Veterinary Parasitology, 248, 68-73.

13. Jiang Y, Tao W, Wan Q, Li Q, Yang Y, Lin Y, Zhang S, Li W. 2015. Zoonotic and potentially host-adapted Enterocytozoon Bieneusi genotypes in sheep and cattle in northeast china and an increasing concern about the zoonotic importance of previously considered ruminant-adapted genotypes. Applied \& Environmental Microbiology, 81(10), 3326-3335.

14. Juránková J, Kamler M, Kovařčík K, Koudela B. 2013. Enterocytozoon bieneusi in Bovine Viral Diarrhea Virus (BVDV) infected and noninfected cattle herds. Research in Veterinary Science, 94(1), 100-104.

15. Karim MR, Dong H, Li T, Yu F, Li D, Zhang L, Li J, Wang R, Li S, Li X, Rume FI, Ning C. 2015. Predomination and new genotypes of Enterocytozoon bieneusi in captive nonhuman primates in zoos in China: high genetic diversity and zoonotic significance. PLoS One, 10(2), e0117991.

16. Kord-Sarkachi E, Tavalla M, Beiromvand M. 2018. Molecular diagnosis of microsporidia strains in slaughtered cows of southwest of Iran. Journal of Parasitic Diseases, 42(1), 81-86.

17. Lee JH. 2007. Prevalence and molecular characteristics of Enterocytozoon bieneusi in cattle in Korea. Parasitology Research, 101(2), 391-396.

18. Leelayoova S, Subrungruang I, Suputtamongkol Y, Worapong J, Petmitr PC, Mungthin M. 2006. Identification of genotypes of Enterocytozoon bieneusi from stool samples from human immunodeficiency virus-infected patients in Thailand. Journal of Clinical Microbiology, 44(8), 3001-3004.

19. Li J, Luo N, Wang C, Qi M, Cao J, Cui Z, Huang J, Wang R, Zhang L. 2016. Occurrence, molecular characterization and predominant genotypes of Enterocytozoon bieneusi in dairy cattle in Henan and Ningxia, China. Parasites \& Vectors, 9, 142.

20. Li W, Feng Y, Santin M. 2019. Host specificity of Enterocytozoon bieneusi and public health implications. Trends in Parasitology, 35(6), 436-451.

21. Liu H, Jiang Z, Yuan Z, Yin J, Wang Z, Yu B, Zhou D, Shen Y, Cao J. 2017. Infection by and genotype characteristics of Enterocytozoon bieneusi in HIV/AIDS patients from Guangxi Zhuang autonomous region,China. BMC Infectious Diseases, $17(1), 684$
22. Lobo ML, Xiao L, Cama V, Stevens T, Antunes F, Matos O. 2006. Genotypes of Enterocytozoon bieneusi in mammals in Portugal. Journal of Eukaryotic Microbiology, 53(Suppl 1), S61-S64.

23. Ma J, Li P, Zhao X, Xu H, Wu W, Wang Y, Guo Y, Wang L, Feng Y, Xiao L. 2015. Occurrence and molecular characterization of Cryptosporidium spp. and Enterocytozoon bieneusi in dairy cattle, beef cattle and water buffaloes in China. Veterinary Parasitology, 207(3-4), 220-227.

24. Matos O, Lobo ML, Xiao L. 2012. Epidemiology of Enterocytozoon bieneusi infection in humans. Journal of Parasitology Research, 2012, 981424.

25. Mirjalali H, Mirhendi H, Meamar AR, Mohebali M, Askari Z, Mirsamadi ES, Rezaeian M. 2015. Genotyping and molecular analysis of Enterocytozoon bieneusi isolated from immunocompromised patients in Iran. Infection, Genetics and Evolution, 36, 244-249.

26. Qi M, Jing B, Jian F, Wang R, Zhang S, Wang H, Ning C, Zhang L. 2017. Dominance of Enterocytozoon bieneusi genotype $\mathrm{J}$ in dairy calves in Xinjiang, Northwest China. Parasitology International, 66(1), 960-963.

27. Rinder H, Thomschke A, Dengjel B, Gothe R, Löscher T, Zahler M. 2000. Close genotypic relationship between Enterocytozoon bieneusi from humans and pigs and first detection in cattle. Journal of Parasitology, 86(1), 185-188.

28. Sak B, Brady D, Pelikánová M, Květoňová D, Rost M, Kostka M, Tolarová V, Hůzová Z, Kváč M. 2011. Unapparent microsporidial infection among immunocompetent humans in the Czech Republic. Journal of Clinical Microbiology, 49(3), 1064-1070.

29. Sak B, Kváč M, Kučerová Z, Květoňová D, Saková K. 2011. Latent microsporidial infection in immunocompetent individuals - a longitudinal study. PLoS Neglected Tropical Diseases, 5 (5), e1162.

30. Santín M, Fayer R. 2009. Enterocytozoon bieneusi genotype nomenclature based on the internal transcribed spacer sequence: a consensus. Journal of Eukaryotic Microbiology, 56(1), 34-38.

31. Santín M, Fayer R. 2009. A longitudinal study of Enterocytozoon bieneusi in dairy cattle. Parasitology Research, 105(1), 141-144.

32. Santín M, Trout JM, Fayer R. 2005. Enterocytozoon bieneusi genotypes in dairy cattle in the eastern United States. Parasitology Research, 97(6), 535-538.

33. Santín M, Dargatz D, Fayer R. 2012. Prevalence and genotypes of Enterocytozoon bieneusi in weaned beef calves on cow-calf operations in the USA. Parasitology Research, 110(5), 20332041.

34. Shi K, Li M, Wang X, Li J, Karim MR, Wang R, Zhang L, Jian F, Ning C. 2016. Molecular survey of Enterocytozoon bieneusi in sheep and goats in China. Parasites \& Vectors, 9, 23.

35. Sulaiman IM, Bern C, Gilman R, Cama V, Kawai V, Vargas D, Ticona E, Vivar A, Xiao L. 2003. A molecular biologic study of Enterocytozoon bieneusi in HIV-infected patients in Lima, Peru. Journal of Eukaryotic Microbiology, 50(Suppl), 591-596.

36. Sulaiman IM, Fayer R, Yang C, Santin M, Matos O, Xiao L. 2004. Molecular characterization of Enterocytozoon bieneusi in cattle indicates that only some isolates have zoonotic potential. Parasitology Research, 92(4), 328-334.

37. Tang C, Cai M, Wang L, Guo Y, Li N, Feng Y, Xiao L. 2018. Genetic diversity within dominant Enterocytozoon bieneusi genotypes in pre-weaned calves. Parasites \& Vectors, 11(1), 170.

38. Tao WF, Ni HB, Du HF, Jiang J, Li J, Qiu HY, Ye L, Zhang XX. 2020. Molecular detection of Cryptosporidium and Enterocytozoon bieneusi in dairy calves and sika deer in four 
provinces in Northern China. Parasitology Research, 119(1), $105-114$

39. Udonsom R, Prasertbun R, Mahittikorn A, Chiabchalard R, Sutthikornchai C, Palasuwan A, Popruk S. 2019. Identification of Enterocytozoon bieneusi in goats and cattle in Thailand. BMC Veterinary Research, 15(1), 308.

40. Valenčáková A, Danišová O. 2019. Molecular characterization of new genotypes Enterocytozoon bieneusi in Slovakia. Acta Tropica, 191, 217-220.

41. Wang L, Zhang H, Zhao X, Zhang L, Zhang G, Guo M, Liu L, Feng Y, Xiao L. 2013. Zoonotic Cryptosporidium species and Enterocytozoon bieneusi genotypes in HIV-positive patients on antiretroviral therapy. Journal of Clinical Microbiology, 51(2), 557-563.

42. Wang R, Li N, Jiang W, Guo Y, Wang X, Jin Y, Feng Y, Xiao L. 2019. Infection patterns, clinical significance, and genetic characteristics of Enterocytozoon bieneusi and Giardia duodenalis in dairy cattle in Jiangsu, China. Parasitology Research, 118(10), 3053-3060.

43. Wang HY, Qi M, Sun MF, Li DF, Wang RJ, Zhang SM, Zhao JF, Li JQ, Cui ZH, Chen YC, Jian FC, Xiang RP, Ning CS, Zhang LX. 2019. Prevalence and population genetics analysis of Enterocytozoon bieneusi in dairy cattle in China. Frontiers in Microbiology, 10, 1399.

44. Weiss LM, Becnel JJ. 2014. Epidemiology of microsporidian human infections, in Microsporidia: Pathogens of Opportunity, Weiss LM, Becnel JJ, Editors, 1st ed. John Wiley \& Sons Inc: Chichester.

45. Yang J, Song M, Wan Q, Li Y, Lu Y, Jiang Y, Tao W, Li W. 2014. Enterocytozoon bieneusi genotypes in children in Northeast China and assessment of risk of zoonotic transmission. Journal of Clinical Microbiology, 52(12), 43634367.

46. Yu F, Qi M, Zhao Z, Lv C, Wang Y, Wang R, Zhang L. 2019. The potential role of synanthropic rodents and flies in the transmission of Enterocytozoon bieneusi on a dairy cattle farm in China. Journal of Eukaryotic Microbiology, 66(3), 435-441.
47. Zhang X, Wang Z, Su Y, Liang X, Sun X, Peng S, Lu H, Jiang N, Yin J, Xiang M, Chen Q. 2011. Identification and genotyping of Enterocytozoon bieneusi in China. Journal of Clinical Microbiology, 49(5), 2006-2008.

48. Zhang Y, Koehler AV, Wang T, Haydon SR, Gasser RB. 2019. Enterocytozoon bieneusi genotypes in cattle on farms located within a water catchment area. Journal of Eukaryotic Microbiology, 66(4), 553-559.

49. Zhang Q, Zhang Z, Ai S, Wang X, Zhang R, Duan Z. 2019. Cryptosporidium spp., Enterocytozoon bieneusi, and Giardia duodenalis from animal sources in the Qinghai-Tibetan Plateau Area (QTPA) in China. Comparative Immunology, Microbiology and Infectious Diseases, 67, 101346.

50. Zhao W, Zhang W, Yang F, Zhang L, Wang R, Cao J, Shen Y, Liu A. 2015. Enterocytozoon bieneusi in dairy cattle in the Northeast of China: genetic diversity of its gene and evaluation of zoonotic transmission potential. Journal of Eukaryotic Microbiology, 62(4), 553-560.

51. Zhao W, Zhou H, Jin H, Sun L, Li P, Liu M, Qiu M, Xu L, Li F, Ma T, Wang S, Yin F, Li L, Cui X, Chan JF, Lu G. 2020. Genotyping of Enterocytozoon bieneusi among captive longtailed macaques (Macaca fascicularis) in Hainan Province: high genetic diversity and zoonotic potential. Acta Tropica, 201, 105211.

52. Zhao W, Zhou H, Yang L, Ma T, Zhou J, Liu H, Lu G, Huang H. 2020. Prevalence, genetic diversity and implications for public health of Enterocytozoon bieneusi in various rodents from Hainan Province, China. Parasites \& Vectors, 13(1), 438.

53. Zhou HH, Zheng XL, Ma TM, Qi M, Cao ZX, Chao Z, Wei LM, Liu QW, Sun RP, Wang F, Zhang Y, Lu G, Zhao W. 2019. Genotype identification and phylogenetic analysis of Enterocytozoon bieneusi in farmed black goats (Capra hircus) from China's Hainan Province. Parasite, 26, 62.

54. Zhou HH, Zheng XL, Ma TM, Qi M, Zhou JG, Liu HJ, Lu G, Zhao W. 2020. Molecular detection of Enterocytozoon bieneusi in farm-raised pigs in Hainan Province, China: infection rates, genotype distributions, and zoonotic potential. Parasite, 27, 12.

Cite this article as: Zheng X-L, Zhou H-H, Ren G, Ma T-M, Cao Z-X, Wei L-M, Liu Q-W, Wang F, Zhang Y, Liu H-L, Xing M-P, Huang L-I, Chao Z \& Lu G. 2020. Genotyping and zoonotic potential of Enterocytozoon bieneusi in cattle farmed in Hainan Province, the southernmost region of China. Parasite 27, 65 .

\section{PARASTE}

An international open-access, peer-reviewed, online journal publishing high quality papers on all aspects of human and animal parasitology

Reviews, articles and short notes may be submitted. Fields include, but are not limited to: general, medical and veterinary parasitology; morphology, including ultrastructure; parasite systematics, including entomology, acarology, helminthology and protistology, and molecular analyses; molecular biology and biochemistry; immunology of parasitic diseases; host-parasite relationships; ecology and life history of parasites; epidemiology; therapeutics; new diagnostic tools.

All papers in Parasite are published in English. Manuscripts should have a broad interest and must not have been published or submitted elsewhere. No limit is imposed on the length of manuscripts.

Parasite (open-access) continues Parasite (print and online editions, 1994-2012) and Annales de Parasitologie Humaine et Comparée (1923-1993) and is the official journal of the Société Française de Parasitologie. 